\title{
Capacidad de gestión para resultados (CGPR) en las organizaciones. El caso de las instituciones públicas mexicanas
}

\author{
Dr. Adolfo Antonio De la Parra Northon \\ adolfodelaparra@gmail.com \\ https://orcid.org/0000-0001-7549-5093 \\ Universidad Juárez del Estado de Durango \\ Dr. Ernesto Geovani Figueroa González \\ geovani.figueroa@ujed.mx \\ https://orcid.org/0000-0002-7900-9141 \\ Universidad Juárez del Estado de Durango
}

\section{RESUMEN}

Las administraciones públicas en el actual proceso de globalización económica buscan mejorar su operatividad mediante aprendizaje para potenciar capacidades de gobiernos con resultados efectivos y crear prosperidad y desarrollo. Un enfoque utilizado con estos fines en México es el modelo Gestión para Resultados (GpR), que basa sus resultados en políticas, programas y proyectos que ejecutan las administraciones públicas. Aunque existen diseños y metodologías desarrolladas por el BID a nivel nacional y subnacional es menester conocer la CPGR en instituciones públicas mexicanas. El presente trabajo pretende mostrar por medio del diseño de un instrumento (encuesta) y su implementación en un estudio de caso la capacidad de Gestión para Resultados (CGpR) en una institución pública mexicana, en este caso la Universidad Juárez del Estado de Durango (UJED). Se delimitan tres dimensiones y se define una estrategia que en base a una escala Likert arroja luz para mejorar sus prácticas con base al modelo de GpR. Se llega a conclusiones y se hacen recomendaciones para su implementación en instituciones públicas mexicanas.

Palabras clave: gestión para resultados; capacidades organizacionales; organizaciones públicas. 


\title{
Management capacity for results (CGPR) in organizations. The case of Mexican public institutions
}

\begin{abstract}
In the current process of economic globalization, public administrations seek to improve their operations through learning to enhance government capacities with effective results and to create prosperity and development. One approach used for these purposes in Mexico is the Management for Results (GpR) model, which bases its results on policies, programs and projects that are executed by public administrations. Although there are designs and methodologies developed by the IDB at the national and subnational level, it is necessary to know the CPGR in Mexican public institutions. The present work aims to show, through the design of an instrument (survey) and its implementation in a case study, the capacity of Management for Results (CGpR) in a Mexican public institution, in this case the Universidad Juarez del Estado de Durango (UJED). Three dimensions are delimited and a strategy is defined which, based on a Likert scale, sheds light to improve their practices based on the GpR model. Conclusions are reached and recommendations are made for its implementation in Mexican public institutions.
\end{abstract}

Keywords: management for results; organizational capabilities; public organizations.

Artículo recibido: 05 octubre. 2021 Aceptado para publicación: 02 noviembre 2021 Correspondencia: geovani.figuero@yahoo.es Conflictos de Interés: Ninguna que declarar 


\section{INTRODUCCIÓN}

El presente trabajo surge de una investigación desarrollada en una institución pública Mexicana de Educación Superior (UJED), como resultado de esta se diseña y aplica un instrumento (encuesta) para medir la capacidad de gestión para resultados (CGPR) en las organizaciones públicas mexicanas.

La estructura general del presente documento plantea el problema, su justificación en torno al contexto de la gestión en las instituciones públicas, un marco teórico y contextual, hipótesis, metodología y análisis de los resultados, conclusiones y recomendaciones al sujeto de estudio. Los instrumentos desarrollados por el Banco Interamericano de Desarrollo (BID) miden el grado de implementación de la GpRD a nivel nacional y subnacional, sin embargo, es menester considerar que el entorno operativo de los gobiernos se realiza por medio de las instituciones públicas, que son realmente quienes se encuentran en contacto con la comunidad. No se contaba con un instrumento que permitiera analizar la capacidad interna de las propias instituciones públicas en cuanto a su GpR, se decidió diseñarlo y aplicarlo en la Universidad Juárez del Estado de Durango (UJED) como organismo público autónomo para la Educación Superior de la Ciudad de Durango.

El objetivo general de la investigación fue medir la Capacidad de Gestión para Resultados (CGpR) en la UJED, por medio de un instrumento que midiera las dimensiones que conforman el término. Ello permitió estructurar una propuesta aportando un instrumento metodológico que sirvió de referente para evaluar la CGpR en instituciones gubernamentales.

Se delimitaron tres dimensiones que dan forma a dicha capacidad: La Coherencia Estratégica (CoE), la Metodología Presupuestaria (MeP) y la del Sistema de información y Gestión ( $\mathrm{SiG}$ ), las cuales se consolidan también por un subdimensionamiento que da forma a los ítems del instrumento que las mide. En el artículo se explica la metodología y se analizan los resultados arrojados, el instrumento se aplica en la Facultad de Economía, Contaduría y Administración (FECA), una de las Facultades de la UJED con mayor matrícula, académicos, administrativos y cuerpo directivo.

Las conclusiones describen como la hipótesis principal se cumplió, pues se pudo construir un instrumento para medir la Capacidad de Gestión para Resultados (CGpR) 
mediante la delimitación de las dimensiones teóricas antes descritas. En cuanto a la hipótesis específica: en H1se desarrolló un instrumento que midió la CGpR en la FECA, y permitió realizar una serie de recomendaciones a la organización para mejorar sus prácticas en torno a la Gestión para resultados (GpR).

En coherencia a lo anterior, el problema a estudiar se centró en encontrar las características que describan la Capacidad de Gestión para Resultados (CGpR) en las organizaciones a partir del diseño y aplicación de un instrumento de medición (encuesta). Se diseño y aplicó la encuesta, se considerarán las dimensiones a analizar aplicadas los administradores, trabajadores y usuarios de los productos y servicios de la UJED, tomando de referencia alguna de sus escuelas, facultades y unidades centrales se desarrollará del Programa Operativo Anual (POA) bajo los criterios de la GpR.

El problema planteado versó entre la CGpR y las variables dimensionales que la conforman, por lo que se utilizó una metodología deductiva para delimitarlas y así, determinar la correlación entre ellas, es decir; se deberá construyó un instrumento que permitió medir la CGpR en una organización y se investigó el grado de correlación entre sus dimensiones. Lo que dio origen a la siguiente pregunta:

« ¿Es posible desarrollar un instrumento basado en aspectos bien delimitados de las organizaciones para determinar su Capacidad de Gestión para Resultados (CGpR)?»

Al profundizar la investigación sobre el marco teórico y el propio objeto de estudio permitió responder a otras interrogantes a resolver en la investigación:

1. ¿Cuáles son las dimensiones que determinan la CGpR en las organizaciones?

2. ¿Cuál es el grado de capacidad entre dichas dimensiones en la FECA?

La respuesta a estas interrogantes permitió contar con un instrumento para poder medir la CGpR en las instituciones de la administración pública y así, construir estrategias que permitan una mayor consolidación de la Gestión para Resultados para el Desarrollo (GpRD) en los gobiernos.

El estudio permitió hacer una contribución metodológica y conceptual mediante un instrumento con base a dimensiones de la CGpR en las instituciones, lo que arrojó resultados de campo que conllevaron a conclusiones sobre la implementación de sistemas administrativos coherentes y efectivos de las organizaciones públicas. 


\section{A partir de los antecedentes señalados se definieron:}

\section{Objetivo General}

- Desarrollar por medio de una metodología teórica deductiva las dimensiones que delimite la Capacidad de Gestión para Resultados (CGpR) en las organizaciones (UJED) y un instrumento que permita medirla, aplicándolo en la FECA como sujeto de estudio.

\section{Objetivos Particulares}

1. Definir mediante un estudio teórico lo que es la CGpR en las organizaciones.

2. Delimitar las dimensiones que permitan medir la CGpR en las organizaciones.

3. Elaborar un instrumento que permita medir la capacidad de gestión para resultados CGpR en la UJED-FECA.

Como elemento conceptual se define la Nueva Gestión Pública (NGP) que consiste, en un primer acercamiento, de acuerdo con Pollitt y Bouckaert (como se citó en Aguilar, 2006, pág. 146): «en cambios deliberados en las estructuras y los procesos de las organizaciones del sector público con el objetivo de que funcionen mejor».

\section{Por su parte, Aguilar la define como:}

«El cambio de las normas, las estructuras organizacionales y los patrones directivos y operativos del gobierno, que por sus propósitos, modos o resultados se orienta hacia formas postburocráticas de organización, dirección y operación, con el fin de elevar la eficiencia, la calidad y la responsabilidad de la acción pública.»

Estos aspectos relacionados con la Nueva Gestión Pública (NGP) lleva implícito la aplicación de modelos como la Gestión para Resultados $(\boldsymbol{G p R})$ para mejorar la gestión de instituciones públicas y su impacto en ls comunidades.

«La Gestión para resultados (GpR), es un modelo de gestión que orienta a los administradores de recursos públicos a poner mayor énfasis en los impactos que genera la intervención pública en los distintos sectores de la sociedad. Como todo modelo de gestión la GpR se integra por una serie de elementos conceptuales, jurídicos e instrumentales que son indispensables para garantizar su operación». 
Se verifica el campo teórico de referencia que dará origen a las dimensiones que conformarán el modelo para medir la CGpR en las organizaciones, pues ésta dependerá de los aspectos principales que aborda la propia GpR de acuerdo a los diferentes autores.

\section{Según Serra (2007) esta lógica comparte, tres objetivos principales:}

1. Asegurar la constante optimización del uso de los recursos públicos en la producción y distribución de bienes.

2. Asegurar que el proceso de producción de bienes y servicios públicos, sean transparentes, equitativas y controlables.

3. Promover y desarrollar mecanismos internos para mejorar el desempeño de los dirigentes y trabajadores públicos, y con ello promover la efectividad de los organismos públicos.

El término GpR ha recorrido un largo camino y es una de las variantes de la Gestión Pública. La GpR se usó principalmente en el sector privado. Aunque el gobierno federal de los Estados Unidos comenzó a utilizar algunos de sus planteamientos en el manejo de diferentes organismos públicos, sólo durante la presidencia de Nixon, a partir de lo que se conoce como la NGP, empezó a implantarse en el conjunto de la administración pública. «Esta nueva filosofía sugiere el paso de una gestión burocrática a una de tipo gerencial. Si bien existen muchos documentos que tratan sobre la GpR, no hay una definición única. La mayoría de los textos usan este término como una noción paraguas».

El objetivo último de la GpR en el sector público es generar capacidad en sus organizaciones para que logren, mediante la gestión del proceso de creación de valor público, los resultados consignados en los objetivos del programa de gobierno. El modelo en sí, tiene la finalidad de mejorar la calidad y la eficacia en la entrega de servicios públicos para enfrentar de mejor manera demandas y tensiones cada vez más complejas y apremiantes, así como rendir cuentas claras a la ciudadanía sobre la administración de los recursos públicos y la efectividad de las acciones.

En este sentido, el Modelo de GpR en el Sector Público enuncia las características de la GpR:

1. Definición de resultados esperados por el gobierno o institución pública. 
2. Orientación a la mejora de la eficacia, eficiencia, productividad y efectividad del uso de los recursos públicos.

3. Monitoreo de la acción pública a través de sistemas de información.

4. Elevar la calidad en los servicios públicos.

5. Aumentar la responsabilidad, compromiso y capacidad de acción de los servidores públicos.

\section{De esta manera, la GpR busca, de forma definitiva:}

- El aumento en la eficiencia y mejora del desempeño.

- El fortalecimiento de la transparencia.

- La rendición de cuentas.

- La calidad del gasto público.

La creación de valor público se considera el fin último de la $\mathrm{GpR}$, a través de la generación de cambios sociales, como se enuncia enel objetivo de la GpR en el sector público es «generar capacidad en sus organizaciones para que logren, mediante la gestión del proceso de creación de valor público, los resultados consignados en los objetivos del programa de gobierno».

Es imperante tener en cuenta que, en la creación de valor público, de acuerdo con Kaufmann, Saginés, \& García Moreno:

«Los cambios sociales no abarcan solamente aquellos que son materia de la política social, como la salud y la educación, sino que también incluyen los relativos a todas las políticas públicas, como la seguridad ciudadana, la agricultura, el empleo, la administración de justicia, la regulación de los mercados o el ejercicio de los derechos ciudadanos. Todos estos aspectos son creadores de valor público, generan cambios en la sociedad y, por tanto, son materia de la GpRD.»; a lo que en la «D» agregada significa «para el Desarrollo».

Para UNAM y SHCP el proceso que sigue la creación de valor público conforma varias etapas: definición de políticas y estrategias, asignación de presupuesto, producción de bienes y servicios, y evaluación de resultados de la intervención gubernamental.

En la implementación de la GpR participan diversos actores: ciudadanos, poder legislativo, poder ejecutivo, directores, técnicos, empresa privada, evaluadores, 
controladores oficiales y controladores sociales.

Los principios fundamentales de la GpR son armonización y alineación, apropiación, resultados, asociación y desarrollo de la capacidad de Aprendizaje según. El sistema de control cobrará un papel determinante en el logro efectivo de las metas establecidas y puede definirse como:

«Es un sistema que intenta diagnosticar, evaluar y corregir las diferentes áreas de la empresa hasta que ésta sea capaz de utilizar sus recursos para cumplir con su misión corporativa.».

En la GpR, un factor fundamental es el Presupuesto basado en Resultados (PbR). La vinculación entre $\mathrm{GpR}$ y $\mathrm{PbR}$, es vital, «no es posible consolidar la Gestión para Resultados sin Presupuesto basado en Resultados, y viceversa».

Se muestra a continuación la relación entre presupuesto tradicional, y el presupuesto para resultados: la aplicación de insumos y bienes intermedios genera productos cuyo resultado tiene impactos en la economía y la sociedad.

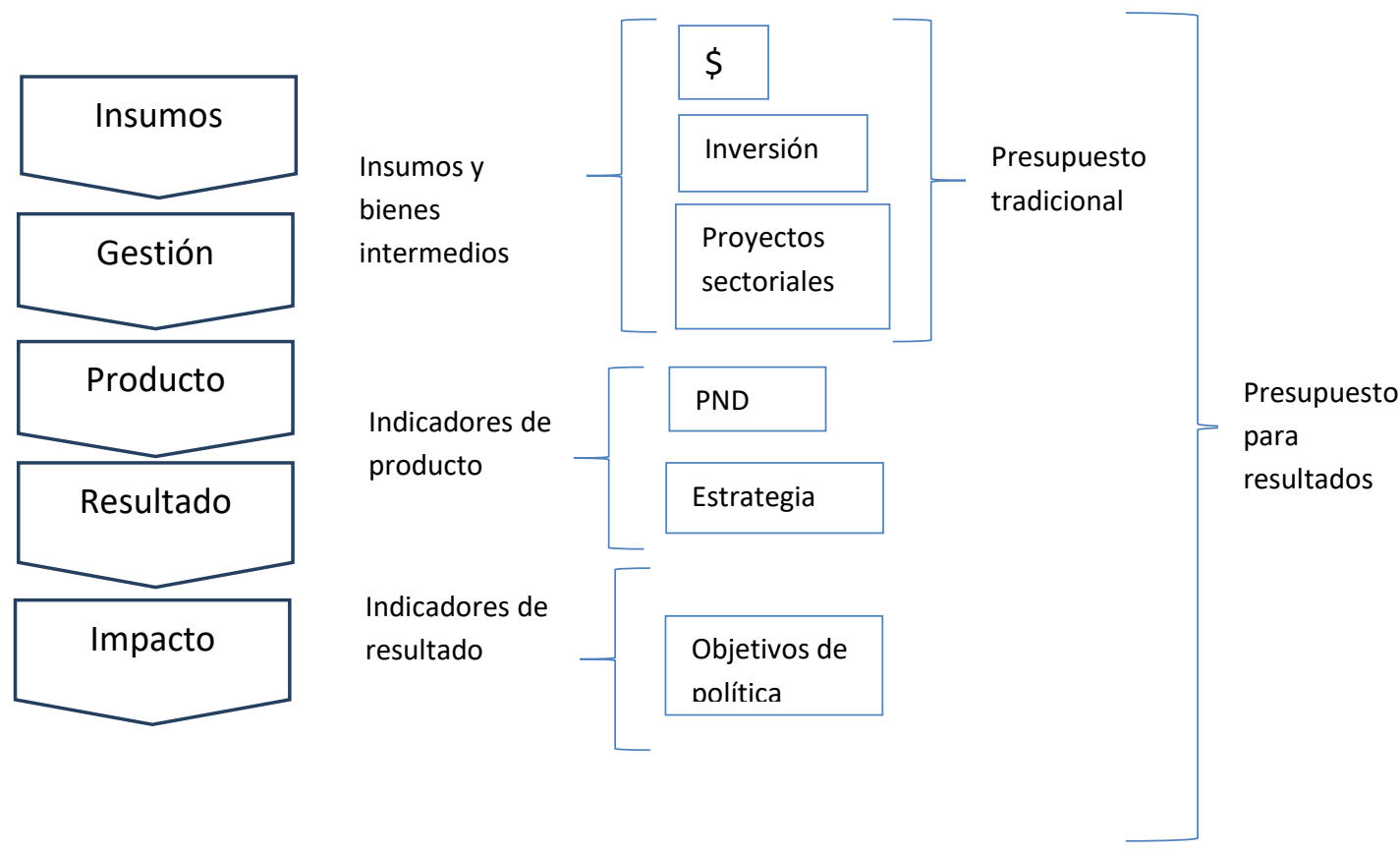

Ilustración 1: Modelo PbR Fuente: Caso (2011).

Es importante que exista motivación en los tomadores de decisiones políticas en la esfera del poder ejecutivo, y fundamental que exista una capacidad administrativa mínima del servicio civil de línea y funcionarios públicos que actúen bajo los incentivos correctos. En lo que concierne al poder Legislativo, su patrocinio de la implementación 
de $\mathrm{PbR}$ es otro de los factores que influye en el éxito de estas reformas. De esta manera, el objetivo principal del $\mathrm{PbR}$ es «aumentar la eficiencia y efectividad del gasto más que el lograr soluciones financieras de corto plazo». Por su parte, es posible reconocer algunos elementos importantes a considerar en la implementación del PbR, dentro de los cuales se subrayan dos: el tamaño del sector público y la presión de la población. Para que haya un equilibrio favorable en el PpR tiene que haber una correspondencia entre el gasto público presupuestado y el ejecutado. En esta dirección, resulta imposible desarrollar el PbR en ausencia de un Sistema de Evaluación del Desempeño que integre Indicadores de Desempeño. Un Sistema de Evaluación del Desempeño se define como:

«...un conjunto de actividades y herramientas que permite apoyar las decisiones presupuestarias; en información que sistemáticamente incorpora consideraciones sobre los resultados del ejercicio de los recursos públicos, y que motiva a las instituciones públicas a lograrlos, con el objeto de mejorar la calidad del gasto, así como la mejora de los bienes y servicios públicos y promover la transparencia y rendición de cuentas».

\section{Las dimensiones del modelo Capacidad de Gestión para Resultados (CGpR) en las organizaciones.}

La Secretaría de Hacienda y Crédito Público, SHCP en su Guía para el Diseño de la Matriz de Indicadores de Resultados (GDMIR), establece que GpR «es un modelo de cultura organizacional, directiva y de desempeño institucional que pone más énfasis en los resultados que en los procedimientos. Aunque también interesa cómo se hacen las cosas, cobra mayor relevancia qué se hace, qué se logra y cuál es su impacto en el bienestar de la población; es decir, la creación de valor público»

\section{Primera dimensión: Coherencia Estratégica $(\mathrm{CoE})$}

En el marco de la GpR, la normatividad de los procesos es primordial, el cual se encuentra desglosado en la presente investigación como la planeación, presupuestación, programación, seguimiento y la evaluación de la planeación estratégica y los programas operativos.

Para Deloitte, un sistema normativo organizacional permite a las organizaciones evolucionar, de esta forma se podrá centrar la atención en la gestión estratégica y la 
vigilancia de la organización. Menciona como sus elementos: Políticas corporativas, Manual de funciones y responsabilidades, Manual de procedimientos, Estructura orgánica, Manual de normas administrativas, Lineamientos de contratación y Código de ética o reglamento laboral.

Comunicar con claridad los objetivos, da certeza a los integrantes de una organización y certeza operativa, puede considerarse parte esencial de donde parte un sistema de control administrativa.

Para Gómez Ceja, los elementos que componen la planeación estratégica deben estar claros y expresados en lenguaje sencillo para facilitar su comprensión. Deberá considerar coherencia con base al proceso de su consolidación, en el sentido de la misión deberá generar certeza de la razón de ser de la organización, la visión deberá estar ligada a ella. Así mismo, el diagnóstico (del análisis FODA) debe definir parámetros para establecer los objetivos estratégicos que darán respuesta a lo detectado en dicho diagnóstico, que representan objetivos delimitados en el mediano y largo plazo, vinculados con la misión que permitirán dar origen a estrategias y líneas de acción preferentes que se instrumentarán (implementación de políticas o programas).

«La planeación estratégica es el proceso de determinar los mayores objetivos de una organización y las políticas y estrategias que gobernarán la adquisición de, uso y disposición de los recursos para realizar esos objetivos».

El proceso de la administración estratégica para Robbins \& Coulter, está conformado de seis pasos, involucra la planeación estratégica (en 4 pasos): 1. Identificar misión actual de la organización, objetivos y estrategias, 2. Análisis externo (Oportunidades y Amenazas), 3. Análisis interno (Fortalezas y Debilidades), 4. Formulación de estratégicas, 5. Implementación de estrategias y 6. Evaluación de resultados.

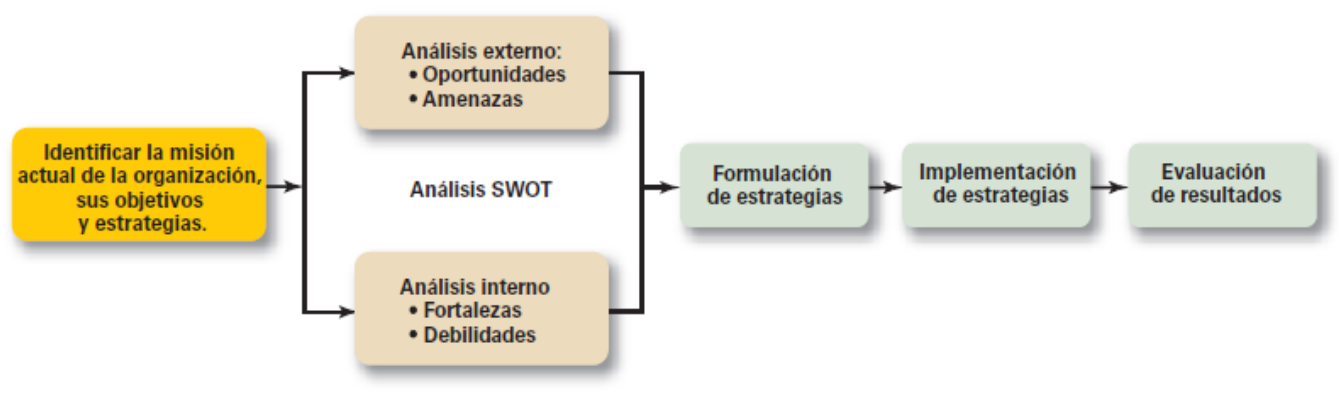

Ilustración 2: El proceso de la administración estratégica Fuente: UNAM y SHCP (2017) 
De acuerdo con la UNAM y SHCP (2017), agregan al procedimiento la visión, quedando un modelo ajustado de 5 pasos: 1. Declaración de la misión y visión, 2. Elaboración del diagnóstico (fortalezas, oportunidades, debilidades y amenazas: FODA), 3. Formulación de objetivos (estratégicos), 4. Definición de estrategias y líneas de acción, y 5. Mecanismos de seguimiento y evaluación.

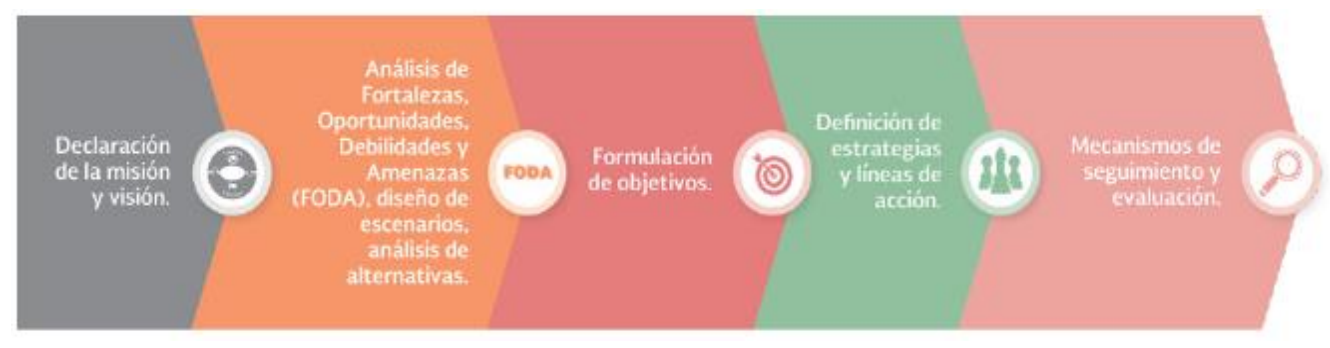

Ilustración3: Planeación estratégica ajustada

La misión y la visión hacen que sea explicito los valores y la direccionalidad general, encuadran, simplifican el espectro de acción y clarifican a los actores en la organización hacia dónde mantener el timón del barco.

\section{Segunda dimensión: Metodología Presupuestaria (MeP)}

Como ya se señaló, la Metodología del Marco Lógico (MML) una herramienta que facilita el proceso de conceptualización, diseño, ejecución, monitoreo y evaluación de programas y proyectos. Por lo tanto, la MeP deberá adoptarla para poder concebir un $\mathrm{PbR}$; es decir una $\mathrm{CGpR}$ más efectiva. Un método puede interpretarse como una secuencia ordenada cronológicamente y eficiente de actividades, que irán a ejecutarse para la obtención de resultados, en este caso los del presupuesto y la planeación estratégica.

La metodología en el sector público ha optado por el Enfoque de Marco Lógico (EML), el cual representa un paso más fino de la planeación estratégica al aplicar un método concreto para la conformación de presupuestos, programas y proyectos. Como bien lo describe Crespo, su extensiva aplicación en diversos contextos permite asumir que sus fortalezas y debilidades están bastante claras y documentadas. 


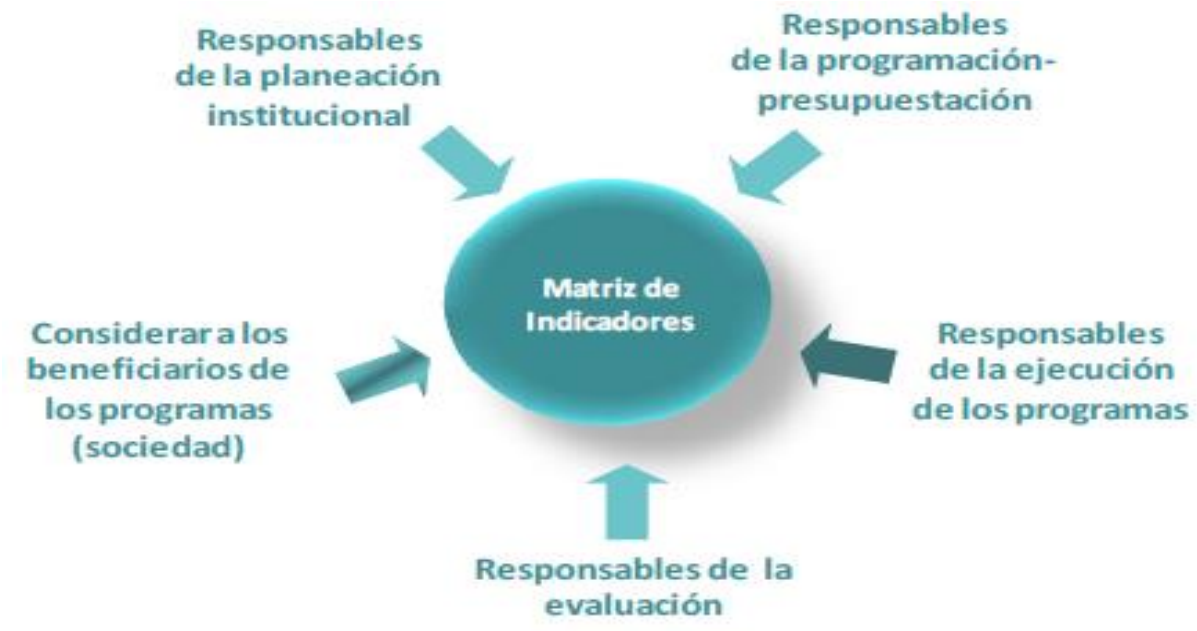

Ilustración4: Principales involucrados en la aplicación de la MML: UNAM y SHCP (2017)

\section{Tercera dimensión: Sistema de información y Gestión (SiG)}

Para la oportuna toma de decisiones y adopción de acciones correctivas por parte de centros de responsabilidad y de la alta dirección, es necesario contar con un sistema de gestión efectivo y debidamente alimentado con información pertinente.

Para Menschel, un sistema es una red de procedimientos relacionados que se desarrollan de acuerdo con un esquema integrado, permite lograr una mayor actividad de la organización. De esta forma de acuerdo con lo establecido y recapitulado por Gómez Ceja contemporáneamente a Menshel lo describe como:

«un conjunto de componentes destinados a lograr un objetivo particular, de acuerdo con un plan... una serie de funciones, actividades $u$ operaciones ligadas entre sí, ejecutadas por un conjunto de empleados para obtener un resultado deseado».

La estructura interna organizacional cobra relevancia, pues el seguimiento depende de una estructura interna adecuada, tal como lo menciona Gómez Ceja:

«La estructura interna e el vehículo mediante el cual la organización extiende su influencia en la sociedad, por tanto, debe invertirse en ella lo suficiente para incrementar $y$ mantener sus capacidades técnicas y sus compromisos con el ambiente».

Es por ello, que un sistema informático bien configurado a las necesidades de desarrollo de una organización, darán una mayor certeza de aprendizaje y de administración de la información, para ello Thompson y Gamble respecto a la instalación de sistemas de 
información refieren: un sistema operativo (a lo que el contexto refiere a un software utilitario) bien pensado, y de vanguardia no sólo permiten ejecutar mejor la estrategia, sino que también fortalecen las capacidades de la organización. Cobrando relevancia en esta instancia sobre la CGpR.

«...contar con un sistema informático que integre los datos sobre los bienes que producen, los servicios que prestan y las obras que realizan los distintos departamentos, programas y proyectos. La idoneidad de estos sistemas de información e indispensable para el buen funcionamiento de una gestión orientada a los resultados y es la base para el sistema de seguimiento y evaluación»

Para la Organización para la cooperación y el Desarrollo Económico (OCDE), la transparencia es un concepto relacionado con la posibilidad de que la información real de una empresa, gobierno u organización puede ser consultada por diferentes sujetos afectados por ella, de tal modo que éstos pueden tomar decisiones con conocimiento de causa y sin asimetría de información.

Es primordial que exista clara cartera de servicios al alcance de usuarios, que éstos hacia el interior puedan ser revisados en cuanto a su efectividad a través de sus indicadores organizacionales. Deben además ser documentos que registren y trasmitan, sin distorsiones, la información básica referente al funcionamiento de las partes del sistema, definición de da Gómez Ceja sobre los manuales de procedimientos.

\section{Entorno organizacional para la operación de la GpR y el desarrollo de sus capacidades}

En México, se ha transitado a una administración para resultados, en base a principios consagrados en la CPEUM en su artículo 134. Los presupuestos públicos ahora deben incluir su impacto real en las comunidades mexicanas, deben contener una serie de elementos esenciales que permitan ser evaluados con un enfoque costo-beneficio.

Como máximo instrumento legal de México también la CPEUM en su Artículo 134 establece que: «Los recursos económicos de que dispongan la Federación, las entidades federativas, los Municipios y las demarcaciones territoriales de la Ciudad de México, se administrarán con eficiencia, eficacia, economía, transparencia y honradez para satisfacer los objetivos a los que estén destinados...» de acuerdo a su párrafo reformado 
en los DOF 07-05-2008, 29-01-2016, entendiéndose «DOF» como el Diario Oficial de la Federación.

En el marco de esta tendencia en la Administración Pública (AP) de los gobiernos, México avanza paulatinamente a una $\mathrm{GpR}$, realiza actos de reforma y modernización en su administración central, marcando el paso a los gobiernos locales para que éstos instruyan los mecanismos y propuestas de modernización para cumplir con los nuevos lineamientos y estándares, logrando mayor desarrollo en las comunidades y un mayor impacto de las políticas públicas en la vida de sus habitantes.

\section{Caso de estudio}

El diseño y aplicación de una metodología para el estudio de Capacidad de gestión para resultados (CGPR) en una institución pública en México se aplicó como muestra en la Facultad de Economía, Contaduría y Administración (FECA) de la Universidad Juárez del Estado de Durango (UJED), la cual tiene bien definida su misión, visión, objetivos estratégicos y es una de las mayores instituciones públicas del Estado de Durango.

\section{Dimensiones de la CGpR}

\section{La Primera dimensión: Coherencia Estratégica (CoE)}

Se puede definir que la $\mathrm{CoE}$ es una correcta y clara estructuración lógica de la planeación y los elementos estratégicos, atendiendo a su vez a aspectos fundamentales:

\section{Lo anterior permite identificar las subdimensiones para la CoE:}

1. Normatividad: Existe la normatividad organizacional para estructurar la GpR en la planeación, presupuestación, programación, seguimiento y evaluación.

2. Claridad: De los elementos que conforman la CoE: misión, visión, plan y/o programa estratégico y sus relaciones causales.

3. Correspondencia: Alineación adecuada entre la misión, visión, diagnóstico (FODA), los objetivos estratégicos.

4. Lógica vertical: En la MIR y la correcta alineación del presupuesto público (Pp) y la Proyección Estratégica (PE).

\section{La Segunda dimensión: Metodología Presupuestaria (MeP)}

Se define que la MeP es la dinámica con la que la organización planea, construye sus programas y los presupuesta. Al considerar dicha metodología se pueden identificar las siguientes subdimensiones para la MeP: 
1. Proceso presupuestario: Los Programas Operativos Anuales (POA) son establecidos con base al Programa de Desarrollo Institucional (PDI) y se conforman mediante un comité o cuerpo directivo que analiza su pertinencia. Se tienen correctamente identificadas todas las fuentes de financiamiento.

2. Consenso programático: Los $\mathrm{Pp}$ se construyen en una dinámica de participación colegiada entre los responsables de planeación, programación, presupuestación, ejecución, evaluación y se hace partícipe a los usuarios de los productos y servicios, es decir; a los beneficiarios.

3. Esquema valorativo: El plan estratégico y los programas operativos anuales, además de su alineación deben contar con metas, objetivos e indicadores que permitan la medición objetiva y congruente de la planeación organizacional.

\section{Tercera dimensión: Sistema de información y Gestión (SiG)}

En un esquema ya más enfocado de la operación de los Pp, se establece el SiG que representa el control efectivo con base a la GpR sobre cada uno de los Pp en los centros de responsabilidad y la alta dirección de la institución.

Atendiendo a lo anterior, se pueden conformar las siguientes subdimensiones para el SiG:

1. Lógica Horizontal: De la MIR construida en el proceso presupuestario: supuestos como externalidades correctamente identificadas, medios de verificación bien identificados y suficientes para los ID y, que éstos describan de manera económica el cumplimiento de cada nivel del resumen narrativo. Al igual que en el PDI por cada objetivo específico.

2. Seguimiento y evaluación: Las diferentes áreas y departamentos de la organización recaban la información en Medios de Verificación (MV) bien definidos en tiempo y forma, realizan los reportes correspondientes mediante formatos específicos y de esta forma alimentan los Indicador(es) de Desempeño (ID) de manera periódica para mantener una retroalimentación actualizada, además, existe un cuerpo directivo bien definido, el cual se reúne periódicamente para analizar los resultados, atender desviaciones y proponer estrategias.

3. Estructura orgánica y procedimental: Debe existir una vinculación adecuada entre las diferentes áreas, específicamente con un centro de responsabilidad que 
reúna y modere la información para la administración de los resultados. Se deben de contar con elementos bien definidos sobre formatos y procesos debidamente formalizados.

4. Sistema de información: Cada unidad administrativa cuenta con el acceso a un sistema de información central que alimenta en lo referente a los Pp que ejecuta, el sistema procesa la información y permite una versión panorámica a la alta dirección y, a las áreas de seguimiento y evaluación para la oportuna toma de decisiones. La información está clara, disponible y trasparente para usuarios internos y externos.

\section{Hipótesis Principal}

- Es factible construir un instrumento para medir la Capacidad de Gestión para Resultados (CGpR) mediante la delimitación de dimensiones teóricas.

\section{Hipótesis específicas}

- H1: Se puede determinar la CGpR de la FECA

- H2: Existe una limitada efectividad de los sistemas de información en la FECA, lo que representará un área de oportunidad para mejorar su CGpR.

\section{Variables independientes}

Permite develar los principales aspectos y elementos que deben esperarse dentro de las organizaciones para considerar una GpR en su desempeño.

\section{Variables dimensionales de dicha capacidad:}

1. Primera dimensión: Coherencia Estratégica (CoE)

\section{Segunda dimensión: Metodología Presupuestaria (MeP)}

3.

4. Tercera dimensión: Sistema de información y Gestión ( $\mathrm{SiG})$

\section{Variable dependiente}

Capacidad de Gestión para Resultados (CGpR): el grado de capacidad en la organización para ejercer una efectiva GpR

\section{ESTRATEGIAS METODOLÓGICAS O MATERIALES Y MÉTODOS}

No se encontró antecedente sobre medición (CGpR) en los organismos públicos y bibliografía consultadas. La investigación tuvo un enfoque cuantitativo no experimental, dado que se otorgarán valores numéricos que permitieron medir el grado con que cuenta la organización y que permita la posibilidad de comparabilidad con otras instituciones. 
El estudio fue exploratorio de orden descriptivo, considerando que el dimensionamiento deberá ser construido desde una base teórica y contextual sobre lo que versa la GpR y deberá ser aplicado en un momento en el tiempo, es decir, la investigación será de corte transversal.

\section{Técnica e instrumento}

Se empleo una técnica que permitió el análisis sobre el objeto de estudio que es la organización, en este caso la FECA de la UJED. Los sujetos fueron las áreas adjetivas de la propia facultad, en el estudio de campo se aplico la encuesta, representando el instrumento mediante el cual se obtenga una medida objetiva sobre dicha capacidad organizacional.

Se aplico la escala de Likert para otorgar valores numéricos al grado de cumplimiento de cada ítem, en donde para cada ítem existe una valoración del grado de acuerdo del sujeto: Totalmente de acuerdo, De acuerdo, Indeciso, En desacuerdo y totalmente en desacuerdo, lo que representa 4, 3, 2, 1 y 0 puntos respectivamente. El total de ítems para el instrumento es de 47, dividido de acuerdo a una tabla, en dónde la máxima puntuación posible es de 188 y la mínima de 0. Ver Anexo 1. Se aplicó en la FECA el cuestionario a todas las áreas, el 100\% de la población.

La estrategia utilizada para la metodología se llevó a cabo de la siguiente manera:

Se conceptualizó la variable a describir; revisión bibliográfica, marco Teórico; dimensiones y subdimensiones a evaluar; operacionalización de las variables dimensionales, diseñó de ítems correspondientes a las subdimensiones; instrumento y sus escalas; se ordenan los ítems y aplicación directa a los sujetos del objeto de estudio; se recopilan datos y se tratan en una base de datos mediante una hoja de cálculo de Microsoft Excel, graficando los resultados; y se valida el instrumento.

\section{RESULTADOS Y DISCUSIÓN}

Al aplicar el instrumento en el sujeto de estudio (FECA), los encuestados evaluaron mediante la escala de Likert y, mediante la proporción simple se puede medir su capacidad sobre cada dimensión y subdimensión para medir la efectividad de la gestión de cada organización.

\section{Resultados de la CGpR en la FECA}


El análisis de forma deductiva plasmo que la CGPR del sujeto de estudio es de $49.79 \%$ representa menos de la mitad de un puntaje perfecto de acuerdo al dimensionamiento, dejando una amplia área de oportunidad del 50.29\%.

El Valor Público (VP) como el fin último de la GpR, en este caso se ocupa en generar capacidad en la organización para que logre, mediante la gestión, los objetivos del Plan de Desarrollo Institucional (PDI) como política estratégica a largo plazo. En síntesis, estos resultados representan una gran área de oportunidad para mejorar la generación de VP y en el control y logro de las políticas estratégicas.

\section{De acuerdo al dimensionamiento primario, en donde se identifican CoE, MeP y SiG,} se dan los siguientes resultados para cada una:

1. Primera dimensión: Coherencia Estratégica $(\mathrm{CoE}): 58.72 \%$

2. Segunda dimensión: Metodología Presupuestaria (MeP): $49.85 \%$

3.

4. Tercera dimensión: Sistema de información y Gestión (SiG): 42.51\%

La mayor área de oportunidad para la FECA es la de SiG. seguido por la MeP y la CoE como su mayor fortaleza, vale mencionar que entre ésta y la $\mathrm{SiG}$ existe un margen de 16.21\%. Valdrá la pena analizar estas variaciones. El escenario que presenta la FECA con estos resultados presentan a la $\mathrm{SiG}$ como la dimensión con mayor área de oportunidad, se hace evidente la falta de concentración, procesamiento y gestión de información mediante tecnologías adecuadas, por otro lado, la CoE presenta la mayor fortaleza, sin embargo, al considerar la puntuación obtenida no es del todo efectiva, representa la correcta y clara estructuración lógica de la planeación y los elementos estratégicos, por así decirlo, la sinergia colaborativa.

Referente a la segunda dimensión, la MeP. Se valida la adecuada aplicación de la Metodología del marco Lógico (MML) para la construcción de la Matriz de Indicadores de Resultados (MIR). En este caso, la FECA no alcanza la mitad de la puntuación mínima en esta dimensión, lo que indica que no se sigue la metodología, la cual puede interpretarse como una secuencia ordenada cronológicamente y eficiente de actividades, que irán a ejecutarse para la obtención de resultados. En el contexto de la FECA, el Programa Operativo Anual (POA), debe construirse mediante la MML y presentado mediante la MIR, en este caso no ejerce con suficiente efectividad el proceso presupuestario. 
La tercera dimensión ( $\mathrm{SiG})$, representa la mayor área de oportunidad, donde obtuvo la menor puntuación, representa el control efectivo en base a la GpR sobre cada uno de los Pp en los centros de responsabilidad y la alta dirección de la institución, los resultados hacen evidente la falta de concentración, procesamiento y gestión de la información mediante tecnologías adecuadas, por tanto, el control y monitoreo de los resultados no se encuentran totalmente disponibles para la toma de decisiones, dificultando de esta forma la adecuada efectividad en el ejercicio de las funciones de las áreas adjetivas y sustantivas del sujeto de estudio.

\section{CONCLUSIÓN O CONSIDERACIONES FINALES}

Atendiendo a los objetivos del estudio, fue posible definir primeramente lo que es la CGpR en las organizaciones como el grado de capacidad en las organizaciones para ejercer una efectiva GpR, considerando para ello tres dimensiones (o pilares) que la constituyen: coherencia estratégica, metodología presupuestaria y sistema de información y gestión, dichas dimensiones se construyeron de acuerdo al contenido deductivo en el marco teórico.

Se encontraron varias coincidencias entre todas las fuentes y autores sobre lo que debe representar la GpR, considerando para la primer dimensión un aspecto de mucho raciocinio práctico, pues se establece la Coherencia Estratégica (CoE) como una correcta y clara estructuración lógica de la planeación y los elementos estratégicos, atendiendo a su vez a tres aspectos fundamentales: la correspondencia entre ellos, la lógica vertical de la MIR y la claridad en la que se expresan dichos elementos debidamente normados.

Para la segunda dimensión, se debe entender que para el desarrollo efectivo de todo programa o proyecto público se tiene que llevar a cabo la MML planeando, construyendo y presupuestando los programas. Es decir, la organización establece su operatividad estratégica y anual de acuerdo con su visión a largo plazo; la Metodología Presupuestaria (MeP)

Finalmente, para la última dimensión que hace referencia a los Sistemas de información y Gestión ( $\mathrm{SiG}$ ) como forma de evaluar y monitorear, imprescindible para la toma de decisiones y para el logro de metas y objetivos, se estableció como aquella que representa el control efectivo con base a la GpR sobre cada uno de los Pp en los centros y la alta dirección de la institución. 
Por lo tanto, la hipótesis principal se cumple, se pudo construir un instrumento para medir la Capacidad de Gestión para Resultados (CGpR) mediante la delimitación de las dimensiones teóricas antes descritas. En cuanto a las hipótesis específicas H1: se desarrolló un instrumento que midió la CGpR en la FECA, y permite realizar una serie de recomendaciones a la organización para mejorar sus prácticas en torno a la $\mathrm{GpR}$, en donde se desvela también el cumplimiento de la H2, donde la FECA muestra su mayor área de oportunidad en sus sistemas de información, en la dimensión «SiG»

\section{Recomendaciones para estudios posteriores}

- Será de ayuda llevar un glosario de términos y abreviaturas para encuestados.

- Aplicar el instrumento propuesto en el resto de las facultades y escuelas de la UJED, organizaciones públicas del Estado de Durango y al resto del país como instrumento eficaz para mejorar la gestión pública.

\section{LISTA DE REFERENCIAS}

Aguilar, L. (2006). Gobernanza y gestión pública. México: Fondo de la Cultura Económica.

Albi Ibáñez, E., González-Páramo, J. M., \& Zubiri, I. (2006). Ecnomía Pública I (Segunda ed.). Barcelona, España: Editorial Ariel.

Aldunate, Eduardo. (2011). Formulación de programas con la metodología de marco lógico (17-26 ed.). Santiago de Chile: instituto latinoamericano del caribe de planeación económica y social.

Arellano Gault, D., Lepore, W., Zamdio, E., \& Blanco, F. (2012). Sistemas de Evaluación del Desempeño para organizaciones públicas. México: CIDE.

Armijo Quintana, M. (2009). Manual de Planificación Estratégica e Indicadores de Desempeño en el Sector Público. Recuperado el 20 de 12 de 2018, de www.cepal.org:

https://www.cepal.org/ilpes/noticias/paginas/3/38453/manual_planificacion_estr ategica.pdf

Armijo, M. (2011). Planficación estratégica e indicadores de desempeño en el sector público. Santiago: CEPAL.

Ayala Espino, J. (2001). Economía del Sector Público Mexicano (Segunda ed.). México: Esfinge. 
Banco Interamericano de Desarrollo. (2012). Gestión para resultados en el desarrollo en gobiernos subnacionales. Washington, D.C., Estados Unidos de America: BID.

Barceleta Chávez, H. (2008). Finanzas Públicas en México 1983-2006 (Primera ed.). Málaga, España: Ediciones Grupo Eumednet.

BID. (Junio de 2011). BID - Instrumento de Diagnóstico - Banco Interamericano de Desarrollo. Recuperado el 21 de Noviembre de 2017, de http://www.iadb.org/es/temas/gobierno/gestion-para-resultados-en-eldesarrollo/instrumento-de-diagnostico, 8360.html

BID. (28 de Mayo de 2018). Banco Interamericano de Desarrollo; Gestión para Resultados en el Desarrollo. Obtenido de https://idblegacy.iadb.org/es/temas/gobierno/gestion-para-resultados-en-eldesarrollo/home,8351.html

Bonnefoy, J. C. (2005). Planificación estratégica en organizaciones públicas. II CursoSeminario Políticas Presupuestarias y Gestión por Resultados. Santiago,Chile.

Calleja Bernal, F. J., \& Calleja, F. (2014). Contabilidad Administrativa. Contabilidad Gerencial. 2a. ed. México: PEARSON.

Caso, A. (2011). La evaluación de las políticas publicas en México. México: INAP.

CEFP, C. d. (2017). www.cefp.gob.mx. Recuperado el 22 de Agosto de 2017, de http://www.cefp.gob.mx/publicaciones/documento/2016/septiembre/cefp023201 6.pdf

Chiavenato, I. (2002). Administración proceso administrativo (3a. ed. ed.). Colombia: McGRAW-HILL INTERAMERICANA, S.A.

Chica, S. (2011). Una mirada a los nuevos enfoques de la gestión pública. Administración y desarrollo, 39(53), 57-74.

CLAD. (Octubre de 1998). Una Nueva Gestión Pública para América Latina; Centro Latinoamericano de Administración para el Desarrollo. Recuperado el 07 de Octubre de 2017, de file:///C:/Users/Secretaria\%20Academica/Downloads/ngpesp\%20(1).pdf

CONEVAL. (2013). Manual para el diseño y la construcción de indicadores. Ciudad de México: CONEVAL. 
CPEUM, C. d. (24 de febrero de 2017). Cámara de Diputados. Recuperado el 23 de agosto de 2017, de http://www.diputados.gob.mx/LeyesBiblio/pdf/1_240217.pdf

CPEUM, C. d. (23 de agosto de 2017). Cámara de Diputados. Recuperado el 23 de agosto de 2017, de http://www.diputados.gob.mx/LeyesBiblio/pdf/1_240217.pdf

Crespo, M. A. (2015). Guía de diseño de proyectos sociales comunitarios bajo el enfoque del Marco Lógico (Segunda ed.). Caracas: eumed.net. Obtenido de www.eumed.net/libros/2009/575

De Andrade Sánchez, F. J. (1985). CPEUM; comentada (Primera ed.). M’xico: UNAM; Universidad Nacional Autónoma de México.

Del Río, C. (2000). El presupuesto: generalidades, tradicional, áreas y niveles de responsabilidad, programas y actividades, base cero, así como teoría y práctica (9a ed.). México: Ediciones Contables, Administrativas y Fiscales.

Dussauge Laguna, M. I. (2016). Gestión para Resultados. México, México: Grupo Editorial Siglo Veintiuno.

Faya Viesca, J. (2008). Finanzas Públicas (Séptima ed.). México: Porrúa.

FECA. (29 de 11 de 2018). feca.ujed.mx. Obtenido de http://feca.ujed.mx/index.html

Fernández Arena, J. (1991). El Proceso administrativo. México: Diana.

Ferra, C. (1995). Evaluación Socioeconómica de Proyectos. Especialización en Preparación y Evaluacion Social de Proyectos. Monterrey: Instituto Tecnológico y de Estudios Superiores de Monterrey.

Filc Gabriel, S. C. (2012). El presupuesto por resultados en América latina, condiciones para su implementación y desarrollo. Departamento de Investigación y Economista: Jefe, 2-13.

Franceschini, F., Galetto, M., \& Maisano, D. (2007). Management by Measurement: Desingning Key Indicators and Performance Measurement Systems. BerlínHeidel-berg: Springer.

Gámiz Parral, M. G. (S/F). Administración Pública. Durango, México: UJED.

García López, R., \& García Moreno, M. (2010). La gestión para resultados en el desarrollo. Avances y desafpios en América Latina y el Caribe. Washington: BID.

García Sánchez, Isabel María. (Mayo de 2007). La nueva gestión pública: evolución y tendencias. Secretaría General de Presupuestos y Gastos, 37-64. 
Gómez Ceja, G. (1994). Planeación y Organización de empresas (Octava ed.). México: McGraww-Hill.

Grupo de Evaluación de la Naciones Unidas, UNEG. (2007). The Role of Evaluation in Results-Based Management.Obtenido de Grupo de Evaluación de la Naciones Unidas, UNEG: http://www.unevaluation.org/document/detail/87

Harmon Michael, M. R. (1999). Teoría de la organización para la administración pública.DF, México: Fondo de Cultura Económica.

Jiménez Castro, W. (1971). Administración pública para el desarrollo integral. México: Fondo de Cultura Económica.

Jiménez González, A. (2014). Curso de Derecho Tributario (Primera ed.). México: Tax Editores Unidos.

Kaufmann, J., Saginés, M., \& García Moreno, M. (2015). Construyendo gobiernos efectivos. Logros y retos de la gestión pública en América Latina y el Caribe. Washington: BID.

Koontz Harold, W. H. (2013). Elementos de Administración (Octava Edición ed.). DF, Mexico: Mc Graw Hill Education.

Koonz, H., \& Weihrich, H. (1998). Administración. Una perspectiva Global (11ed.). México: McGraw-Hill.

LCF, L. d. (17 de julio de 2016). Cámara de Diputados. Recuperado el 06 de septiembre de 2017 , de http://www.diputados.gob.mx/LeyesBiblio/pdf/31_180716.pdf

LDFEFM, C. d. (25 de agosto de 2017). Cámara de Diputados. Recuperado el 25 de agosto de 2017, de http://www.diputados.gob.mx/LeyesBiblio/pdf/LDFEFM_270416.pdf

LFPRH. (28 de noviembre de 2016). Cámara de Diputados. Recuperado el 16 de septiembre de 2017, de http://www.diputados.gob.mx/LeyesBiblio/pdf/59_281116.pdf

LGCG. (18 de julio de 2016). Cámara de Diputados. Recuperado el 23 de Octubre de 2017, de http://www.diputados.gob.mx/LeyesBiblio/pdf/LGCG_180716.pdf

López, R. G., \& Moreno, M. G. (2010). La gestión para resultados en el desarrollo: Avances y desafios en América Latina y el Caribe. Washington, DC, Estados Unidos de America: Banco Interamericano de Desarrollo. 
Martín Arribas, M. C. (2004). Diseño y validación de cuestionarios. Matronas Profesión, V(17), 23-29.

Martner, G. (1967). Planeación y Presupuesto por Programas. México: Siglo XXI.

Merino, M. (2009). Políticas públicas. Ensayo sobre la intervención del Estado en la solución de problemas públicos. Ciudad de México: CIDE.

Meza, O. (2015). Agenda local. El entorno institucional detrás del proceso de las políticas públicas. Ciudad de Mpéxico: Instituto Tecnológico y de Estudios Superiores de Occidente.

Michel, G. (1974). Ecología de la Organización. México: Trillas.

Mouzelis, N. P. (1975). organización y burocracia. Un análisis de las teorías modernas sobre organizaciones sociales. Barcelona, España: Peninsula.

Muñoz Amato, P. (1975). Introducción a La Administración Pública.. D.F., México: Fondo de Cultura Económica.

OCDE. (1995). Indicadores del Sistema Educativo: la medición de la caliad de las escuelas. París: Organización para la Coperación y el Desarrollo Económicos.

OCDE. (2006). Libro de Consulta. En O. p. Económico, Buenas prácticas recientemente identificadas de gestión para resultados de desarrollo (pág. 6).

Ochoa, G. (1996). Administración Financiera. 3a. ed. . México: Alhambra.

OECD, Banco Mundial. (2005). Buenas Prácticas Recientemente Identificadas de Gestion Para Resultados de Desarrollo: Libro de Consulta. Primera. Recuperado el 21 de julio de 2019, de https://www.oecd.org/dac/effectiveness/36853632.pdf

Ormond Derry y Loffler Elke. (febrero de 1999). Nueva Gerencia Pública: ¿que tomar y que dejar? Revista del CLAD Reforma y Democracia., No. 13, 1 - 10.

Penagos, S. (2008). La reconversión de los procesos gubernamentales en México con un enfoque hacia la mejora, el desempeño y los resultados. Revista de la Administración Pública, XLIII(3), 13-29.

Pérez-Jácome Friscione, D. (2012). Presupuesto basado en Resultados. Recuperado el 12 de Enero de 2017, de SHCP: http://www.hacienda.gob.mx/EGRESOS/sitio_pbr/Documents/Pbr_Mex_02072 012.pdf 
Pichardo Pagaza, I. (2002). Introducción a la nueva administración pública de México (Vol. 1). México: Instituto Nacional de Administración Pública, A.C.

Pozzi, S. (30 de septiembre de 2016). 10 empresas más grandes que 180 países. El País, págs. 1-5. Recuperado el 01 de septiembre de 2017, de https://economia.elpais.com/economia/2016/09/29/actualidad/1475150102_4548 18.html

Rea Azpeitia, Elias Alfonso. (Enero-Marzo de 2013). Componentes de la gestión para resultados en los municipios. Hacienda municipal, 66-79.

Robbins, S. P., \& Coulter, M. (2010). Administración (Décima ed.). (L. Fernández Enríquez, \& V. d. Alba Ramírez, Trads.) México, México: PEARSON EDUCACIÓN,.

Rodríguez Lobato, R. (2004). Derecho fiscal (Segunda ed.). México, Edo. de México,

Sanín, H. (07 de Septiembre de 2016). Control de gestión y evaluación de resultados en la gerencia pública. Obtenido de http://unpan1.un.org/intradoc/groups/public/documents/uneclac/unpan014539.pd $\mathrm{f}$

SHCP. (s.f.). Guía para el diseño de la Matriz de Indicadores para Resultados. Ciudad de México: SHCP.

SHCP; Secretaría de Hacienda y Crédito Público. (08 de julio de 2017). Secretaría de Hacienda y Crédito Público | Gobierno | gob.mx. Recuperado el 15 de septiembre de 2017, de https://www.gob.mx/shcp/prensa/comunicado-no-102predial-fuente-de-ingreso-para-fortalecer-finanzas-y-desarrollo-urbano-en-elpais-meade-kuribrena?idiom=es

Stiglitz, J. (2000). La economía del sector público (Tercera ed.). Barcelona, España: Antoni Bosch.

Stoner, J., Freeman R., E., \& Gilbert, D. R. (1996). Administración. México: Prentice Hall.

Tenorio Hinojosa, H. A. (2014). El Sistema Nacional de Coordinación Fiscal en México. Praxis de la Justicia Fiscal y ADministrativa, 6(15), 4-5.

Torres Prada, L. (2001). "Indicadores de gestión para las entidades públicas". Revista Española de Financiación y Contabilidad., vol. XXI, núm. 67, pp. 535-558. 
Capacidad de gestión para resultados...

UJED. (28 de 11 de 2018). www.ujed.mx. Obtenido de https://www.ujed.mx/identidadinstitucional

UNAM y SHCP. (2017). Diplomado de Presupuesto Basado en Resultados (PbR). Diplomado. México, México: Universisdad Nacional Autónoma de México y la Secretaría de Hacienda y Crédito Público.

World Bank. (2018). 3rd High Level Forum On Aid Effectiveness. Managing for Development Results. Accra Ghana. 
Anexo No.1 Encuesta para medir la Capacidad de Gestión para Resultados (CGpR) en las organizaciones.

\begin{tabular}{|c|c|c|}
\hline \multicolumn{3}{|c|}{ Capacidad de Gestión para Resultados (CGpR) en la organización } \\
\hline \multicolumn{3}{|c|}{$\begin{array}{l}\text { Encuesta de aplicación directa a los titulares de las unidades adjetivas y sustantivas de la } \\
\text { organización }\end{array}$} \\
\hline & & Lea cuidadosamente las instrucciones: \\
\hline & 1 & $\begin{array}{l}\text { Para efecto de la presente, considere como "Institución" } \\
\text { únicamente a la FECA; es decir, omita considerar referencias a la } \\
\text { administración central de la UJED. }\end{array}$ \\
\hline & 2 & $\begin{array}{l}\text { Marque a la derecha de la aseveración con el número que } \\
\text { corresponda a su «GRADO DE ACUERDO» en referencia al } \\
\text { centro en el que trabaja y el puesto que desempeña, conforme la } \\
\text { siguiente escala: }\end{array}$ \\
\hline & & Totalmente de acuerdo: 4 \\
\hline & & De acuerdo: 3 \\
\hline & & Indeciso: 2 \\
\hline & & En desacuerdo: 1 \\
\hline & & Totalmente en desacuerdo: 0 \\
\hline No. & Pto. & Ítem \\
\hline 1 & 1.1.1. & $\begin{array}{l}\text { Existe la normatividad necesaria y suficiente, en reglamentos o } \\
\text { normas que garanticen el proceso de planeación, presupuestación } \\
\text { y programación con base a resultados. }\end{array}$ \\
\hline 2 & 1.1.2. & $\begin{array}{l}\text { Existe la normatividad necesaria y suficiente, en reglamentos o } \\
\text { normas que garanticen el ejercicio para el reporte de avances, el } \\
\text { seguimiento y la evaluación. }\end{array}$ \\
\hline 3 & 1.1.3. & $\begin{array}{l}\text { El proceso de la aplicación de la Metodología del Marco Lógico } \\
\text { (MML) para la elaboración de los Programas Operativos Anuales } \\
\text { (POA) se encuentra debidamente reglamentada. }\end{array}$ \\
\hline 4 & 1.2.1. & Conozco y me es clara la Misión organizacional. \\
\hline 5 & 1.2.2. & Conozco y me es clara la Visión organizacional. \\
\hline 6 & 1.2.3. & Conozco y me es claro el Plan de Desarrollo Institucional (PDI). \\
\hline 7 & 1.2.4. & $\begin{array}{l}\text { Conozco los Programas Operativos Anuales (POA's) que me } \\
\text { competen institucionalmente, de forma detallada y comprendo la } \\
\text { menara en la que se vincula y contribuye con el Programa de } \\
\text { Desarrollo Institucional (PDI). }\end{array}$ \\
\hline 8 & 1.3.1. & $\begin{array}{l}\text { Es clara la dirección y certeza que da el Programa de Desarrollo } \\
\text { Institucional (PDI) para alcanzar la Visión institucional. }\end{array}$ \\
\hline
\end{tabular}




\begin{tabular}{|c|c|c|}
\hline 9 & 1.3 .2 & $\begin{array}{l}\text { El (los) Programa Operativo Anual (POA) de su área, impacta en } \\
\text { las metas del Programa de Desarrollo Institucional (PDI). }\end{array}$ \\
\hline 10 & 1.3 .3 & $\begin{array}{l}\text { El (los) Programa Operativo Anual (POA) de su área es coherente } \\
\text { con la Misión organizacional. }\end{array}$ \\
\hline 11 & 1.3 .4 & $\begin{array}{l}\text { Existe una coherencia clara entre la Misión y Visión } \\
\text { organizacional. }\end{array}$ \\
\hline 12 & 1.3 .5 & $\begin{array}{l}\text { Se realizó un diagnóstico organizacional (FODA) participativo } \\
\text { para la construcción del Plan de Desarrollo Institucional (PDI), } \\
\text { tomando en cuenta la Visión. }\end{array}$ \\
\hline 13 & 1.4 .1 & $\begin{array}{l}\text { Las Actividades contempladas en la Matriz de Indicadores de } \\
\text { Resultados (MIR) del (de los) Programa Operativo Anual (POA), } \\
\text { son los suficientes y necesarios para desarrollar los bienes y/o } \\
\text { servicios establecidos. }\end{array}$ \\
\hline 14 & 1.4 .2 & $\begin{array}{l}\text { Los Componentes (bienes y servicios) establecidos en la Matriz de } \\
\text { Indicadores de Resultados (MIR), son los suficientes y necesarios } \\
\text { para hacer cumplir con el Propósito del (de los) Programa } \\
\text { Operativo Anual (POA). }\end{array}$ \\
\hline 15 & 1.4 .3 & $\begin{array}{l}\text { El Propósito del Programa Operativo Anual (POA) contribuye al } \\
\text { cumplimiento del Plan de Desarrollo Institucional (PDI) y/o de su } \\
\text { fuente de financiamiento. }\end{array}$ \\
\hline 16 & 2.1 .1 & $\begin{array}{l}\text { Se reúne el personal de la Unidad Administrativa, o en su caso el } \\
\text { Cuerpo Directivo para definir la Situación Problemática que se } \\
\text { atenderá con el (cada) Programa Operativo Anual (POA) con el } \\
\text { fin de lograr las metas del Plan de Desarrollo Institucional (PDI). }\end{array}$ \\
\hline 17 & 2.1 .2 & $\begin{array}{l}\text { Se consideran a los Involucrados de la Situación Problemática } \\
\text { para medir las Expectativas y Fuerzas de los Actores, de acuerdo a } \\
\text { los escenarios definidos para intervenir con el Programa } \\
\text { Operativo Anual (POA). }\end{array}$ \\
\hline 18 & 2.1 .3 & $\begin{array}{l}\text { Se realiza una adecuada conversión de la Situación Problemática a } \\
\text { atender en una Situación Objetivo, considerando los medios para } \\
\text { alcanzarla. }\end{array}$ \\
\hline 19 & 2.1 .4 & $\begin{array}{l}\text { Se realiza un Análisis de Alternativas de bienes, servicios y sus } \\
\text { actividades, con base a una Matriz que evalúe los Aspectos } \\
\text { Cualitativos de cada Alternativa planteada para seleccionar la } \\
\text { mejor opción. }\end{array}$ \\
\hline 20 & 2.1 .5 & $\begin{array}{l}\text { Con base a la Situación Objetivo del Programa Operativo Anual } \\
\text { (POA), se construye una Matriz de Indicadores de Resultados } \\
\text { (MIR) que contemple Metas, Indicadores, Medios de Verificación } \\
\text { y Supuestos para cada nivel de ésta. }\end{array}$ \\
\hline 21 & 2.1.6. & $\begin{array}{l}\text { Se consideran las Fuentes de Financiamiento alternativas } \\
\text { disponibles para intervenir en la Situación Objetivo, se vinculan } \\
\text { correctamente con el Plan de Desarrollo Institucional (PDI) y/o a } \\
\text { las Políticas Estratégicas de la Fuente de Financiamiento (en su } \\
\text { caso). }\end{array}$ \\
\hline 22 & 2.2 .1 & $\begin{array}{l}\text { Para la elaboración de la Matriz de Indicadores de Resultados } \\
\text { (MIR) usted como responsable, se reúne con los responsables de } \\
\text { la Planeación, Programación y Presupuestación organizacional. }\end{array}$ \\
\hline
\end{tabular}




\begin{tabular}{|c|c|c|}
\hline 23 & 2.2.1. & $\begin{array}{l}\text { Para la elaboración de la Matriz de Indicadores de Resultados } \\
\text { (MIR) usted como responsable, se reúne usted con el responsable } \\
\text { de la evaluación organizacional. }\end{array}$ \\
\hline 24 & 2.2.1. & $\begin{array}{l}\text { Para la elaboración de la Matriz de Indicadores de Resultados del } \\
\text { programa usted sondea a los beneficiarios directos del mismo. }\end{array}$ \\
\hline 25 & 2.3.1. & $\begin{array}{l}\text { La Matriz de Indicadores de Resultados (MIR) contiene Objetivos } \\
\text { Específicos que se medirán con Indicadores de Desempeño. }\end{array}$ \\
\hline 26 & 2.3.2. & $\begin{array}{l}\text { El Plan de Desarrollo Institucional (PDI) contiene Objetivos } \\
\text { Específicos que se medirán con Indicadores de Desempeño. }\end{array}$ \\
\hline 27 & 2.3.3. & $\begin{array}{l}\text { Los Indicadores de Desempeño de la Matriz de Indicadores de } \\
\text { Resultados (MIR) se encuentran valorados objetivamente a través } \\
\text { de una Ficha Técnica que garantizan su pertinencia, las cuales } \\
\text { incluyen: las metas a lograr, las variables que intervienen en su } \\
\text { cálculo y una semaforización del nivel de cumplimiento. }\end{array}$ \\
\hline 28 & 2.3.4. & $\begin{array}{l}\text { Los Indicadores de Desempeño del Plan de Desarrollo } \\
\text { Institucional (PDI) se encuentran valorados objetivamente a través } \\
\text { de una Ficha Técnica que garantizan su pertinencia, las cuales } \\
\text { incluyen: las metas a lograr, las variables que intervienen en su } \\
\text { cálculo y una semaforización del nivel de cumplimiento. }\end{array}$ \\
\hline 29 & 3.1.1. & $\begin{array}{l}\text { Los Medios de Verificación de la Matriz de Indicadores de } \\
\text { Resultados son los suficientes y necesarios para alimentar y } \\
\text { accionar correctamente a los Indicadores de Desempeño. }\end{array}$ \\
\hline 30 & 3.1 .2 & $\begin{array}{l}\text { Existen Medios de Verificación suficientes y necesarios; éstos } \\
\text { alimentan y accionan correctamente los Indicadores de } \\
\text { Desempeño. }\end{array}$ \\
\hline 31 & 3.1 .3 & $\begin{array}{l}\text { Los Indicadores de Desempeño permiten observar de forma clara } \\
\text { y objetiva el avance de cumplimiento del Objetivo Específico para } \\
\text { cada nivel de la Matriz de Indicadores de Resultados (MIR). }\end{array}$ \\
\hline 32 & 3.1.4. & $\begin{array}{l}\text { Los Indicadores de Desempeño del Plan de Desarrollo } \\
\text { Institucional (PDI) permiten observar de forma clara y objetiva el } \\
\text { avance de cumplimiento de cada Objetivo Específico. }\end{array}$ \\
\hline 33 & 3.1 .5 & $\begin{array}{l}\text { Los Supuestos de la Matriz de Indicadores de Resultados en cada } \\
\text { nivel permiten evaluar los riesgos por externalidades no } \\
\text { controlables por la organización en cada nivel de la misma. }\end{array}$ \\
\hline 34 & 3.2 .1 & $\begin{array}{l}\text { Existe un Seguimiento efectivo, es decir; un monitoreo } \\
\text { permanente y periódico de los avances del cumplimiento de las } \\
\text { metas organizacionales establecidas en la Planeación. }\end{array}$ \\
\hline 35 & 3.2.2. & $\begin{array}{l}\text { Existe una Evaluación efectiva, es decir; una verificación } \\
\text { permanente y periódica de los avances del cumplimiento de las } \\
\text { metas organizacionales establecidas en la Planeación. }\end{array}$ \\
\hline 36 & 3.2.3 & $\begin{array}{l}\text { Los reportes de las actividades realizadas y sus impactos se } \\
\text { realizan a través de Tiempos debidamente establecidos. }\end{array}$ \\
\hline 37 & 3.2.4. & $\begin{array}{l}\text { El Cuerpo Directivo (o cuerpo colaborativo del programa) se } \\
\text { reúne periódicamente para Validar los Resultados, Retroalimentar } \\
\text { y Tomar Decisiones en el curso de la ejecución de los programas. }\end{array}$ \\
\hline
\end{tabular}




\begin{tabular}{|c|c|c|}
\hline 38 & 3.3 .1 & $\begin{array}{l}\text { En la Estructura Interna de la organización existe un Área } \\
\text { Responsable específicamente del Monitoreo, Seguimiento y } \\
\text { Evaluación del Ejercicio Programático. }\end{array}$ \\
\hline 39 & 3.3 .2 . & $\begin{array}{l}\text { Realiza los reportes de las actividades realizadas y sus impactos se } \\
\text { realizan a través de formatos debidamente establecidos y } \\
\text { formalizados. }\end{array}$ \\
\hline 40 & 3.3.3. & $\begin{array}{l}\text { Existe un Manual de Procedimientos debidamente formalizado y } \\
\text { funcional. }\end{array}$ \\
\hline 41 & 3.3 .4 & $\begin{array}{l}\text { Existe un Manual de Organización debidamente formalizado y } \\
\text { funcional. }\end{array}$ \\
\hline 42 & 3.4 .1 & $\begin{array}{l}\text { Existe un Catálogo de Bienes y Servicios de la organización bien } \\
\text { definido. }\end{array}$ \\
\hline 43 & 3.4 .2 & $\begin{array}{l}\text { Usted cuenta con un Sistema Informático que le permite consultar } \\
\text { la Información suficiente y pertinente para la Toma de Decisiones } \\
\text { oportuna. }\end{array}$ \\
\hline 44 & 3.4.3. & $\begin{array}{l}\text { Existe un Sistema Informático Automatizado que arroja de forma } \\
\text { Actualizada (por lo menos tres meses) el estado de todos los } \\
\text { Indicadores de Desempeño organizacionales. }\end{array}$ \\
\hline 45 & 3.4 .4 & $\begin{array}{l}\text { Usted utiliza un Sistema de Información central que alimenta los } \\
\text { resultados organizacionales. }\end{array}$ \\
\hline 46 & 3.4 .5 & $\begin{array}{l}\text { Existe una Plataforma de consulta sobre los resultados } \\
\text { organizacionales a corto y largo plazo para los usuarios internos. }\end{array}$ \\
\hline 47 & 3.4 .6 & $\begin{array}{l}\text { Existe una Plataforma de consulta sobre los resultados } \\
\text { organizacionales a corto y largo plazo para los usuarios externos. }\end{array}$ \\
\hline
\end{tabular}

